\title{
البيئة كمجال تعليمي في المدرسة الابتدائية بالجزائر
}

الباحثة: مازيا عيساوي

جامعة بسكرة، الجزائر

الملخص:

يتناول هذا المقال مفهوم الثقافة البيئية كوسيلة لتطوير الوعى بغية بلورة

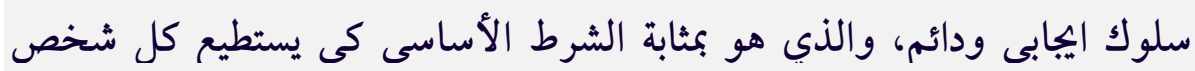
أن يؤدي دوره بشكل فعّال في حماية البيئة وبالتالي المساهمة في الحفاظ عليها ليها. و نظرا لما للثقافة البيئية من أهمية في حياتنا وخاصة الأطفال، أصبح من

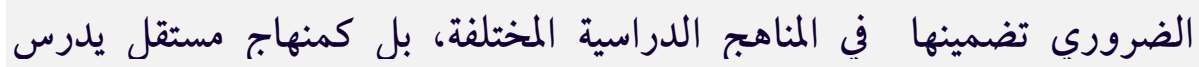

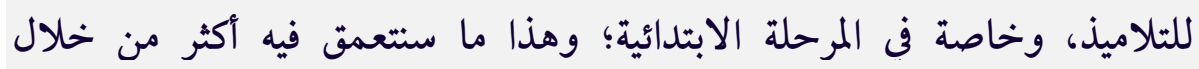

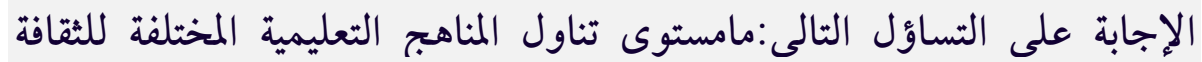
البيئية في المدرسة الجزائرية؟ على الجسول

\section{Résumé :}

Cet article traite la notion de la culture environnementale comme moyen de développement de la conscience environnementale en vue d'acquérir un comportement environnemental positif et durable, ce qui est une condition fondamentale pour que chaque individu puisse jouer efficacement son rôle dans la protection et la préservation de l'environnement. Vu l'importance de la culture environnementale dans notre vie et surtout les enfants, il est urgent et important qu'elle soit incluse dans les différents programmes scolaires et en particulier ceux du primaire. Notre présente étude se focalise sur ce thème en tentant de répondre à la problématique suivante: A quel niveau les différents programmes scolaires en Algérie traitent-ils la culture environnementale? 
خلال العقود الأخيرة تحولت البيئة ومشكلاتها، مع تفاقم تداعياتها

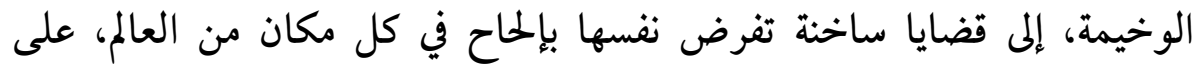

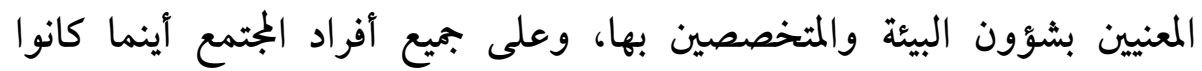

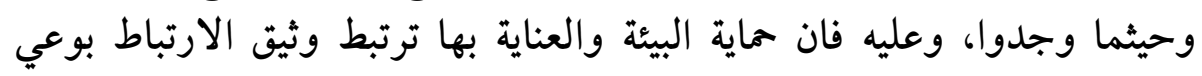
الإنسان وثقافته البيئية.

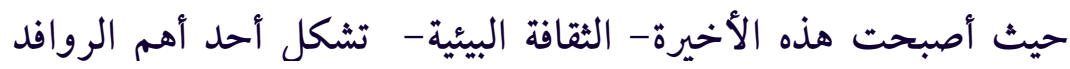

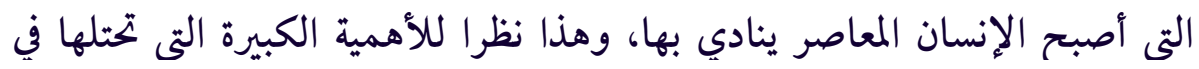

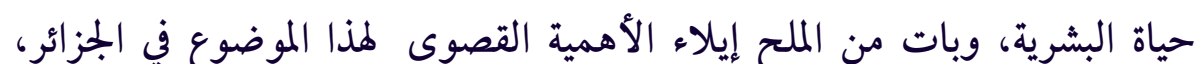

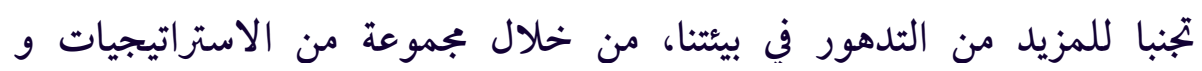
التدابير.

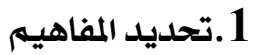

1.1

1.1.1

ثقافة، مصدر ثقف بالضم: صار حاذقا خفيفا فطنا (1). 2.1 تعريف الثقافة اصطلاحا:

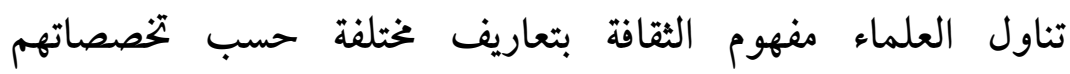

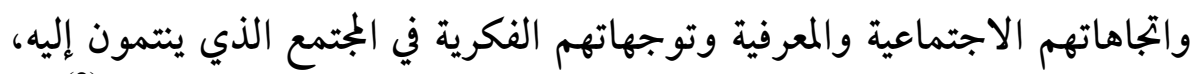

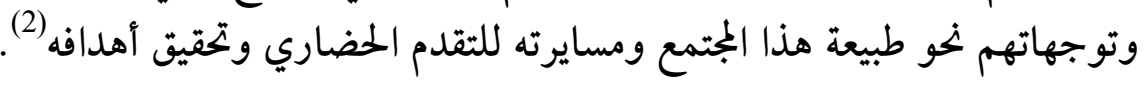

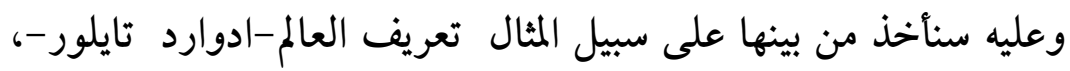

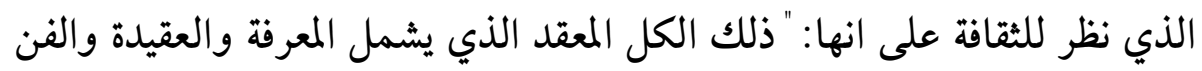

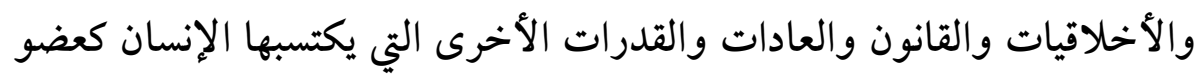
في المجتمع (3) 
يعود الأصل اللغوي لكلمة البيئة في اللغة العربية إلى المصدر" بوآ، الذي

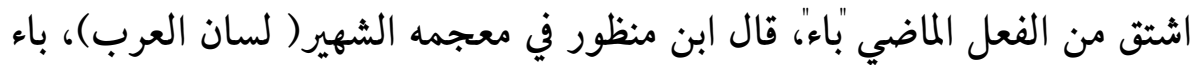

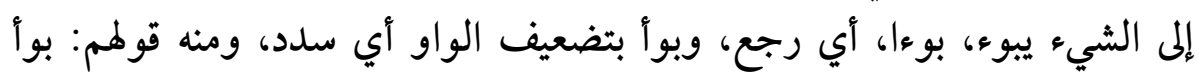

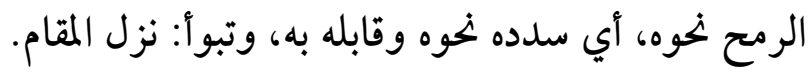

تقول: تبوأ فلان بيتا، أي اتخذ منزلا، وذلك إذا نظر إلى أسهل مايراه وأكثر استواء

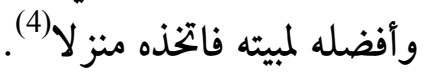

\subsection{1 تعريف البيئة اصطلاحا:}

ولا يختلف المعنى الاصطلاحي للبيئة عن معناها اللغوي كثيرا وعلى مئى

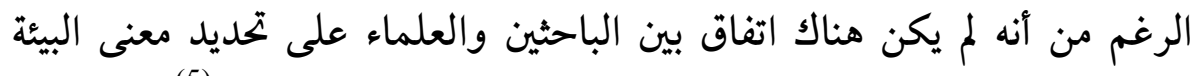

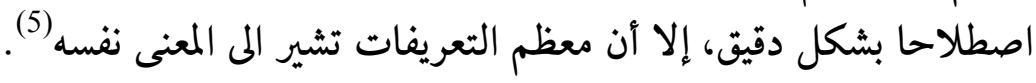
وقد عرف برنامج الأمم المتحدة البيئة على أنها:" الإطار الذي يجيا فيه

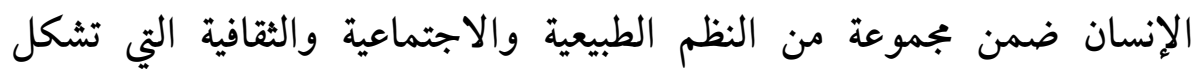
الدورة الحياتية للإِنسان والكائنات الأخرى (6).

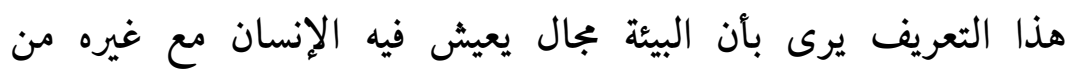

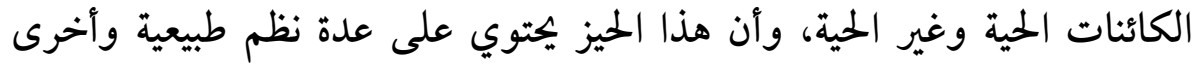

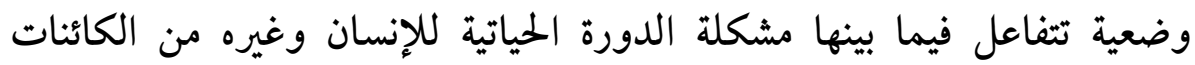
الأخرى (7)

3.1

الثقافة البيئية هي ذلك الكل المركب الذي يشمل المعارف والقيم والاتجاهات البيئية، أي كل ما يتعلق بالبيئة، غير أن الثقافة البيئية ترتبط بالتربية الثية 
والتعليم ولعل ابرز مثال يتحدث عن الثقافة البيئية هو ذلك الذي يربطها بعنصرين هما المشاركة والتعليم البيئي بمعنى السلوك البيئي والوعي البيئي (8). كما عرفت الثقافة البيئية على أنها:" كل مايعبر عن اكتساب الفرد

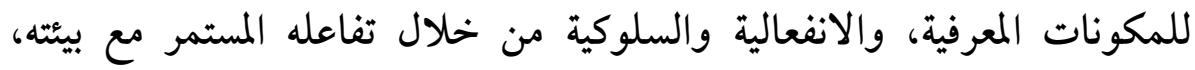

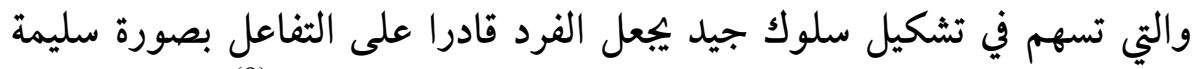

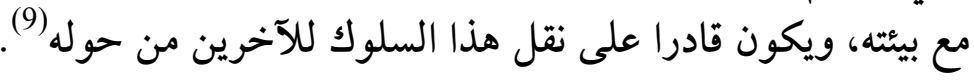

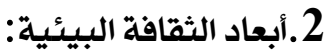

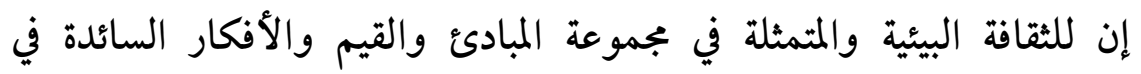

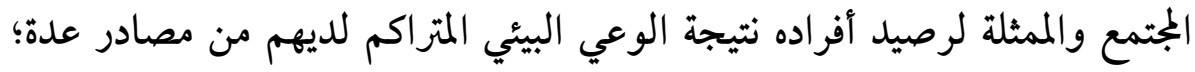

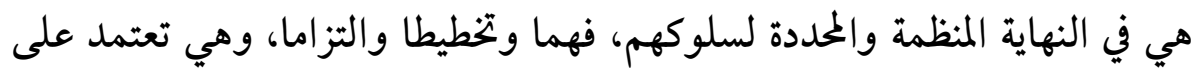
عنصرين أساسيين:

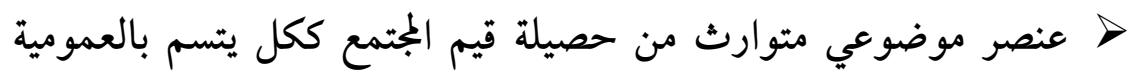

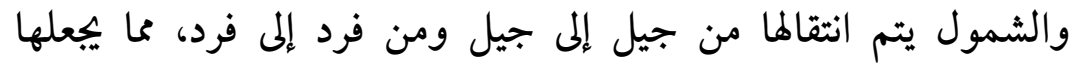

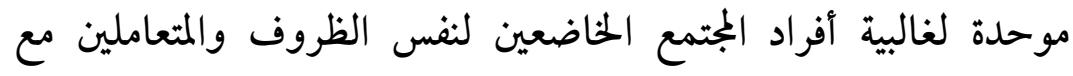
نفس المعطيات.

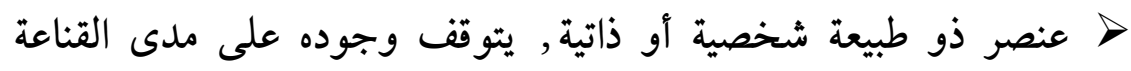

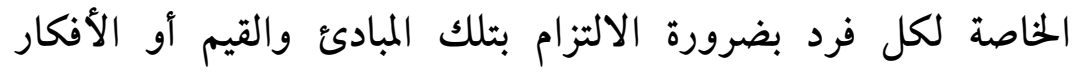

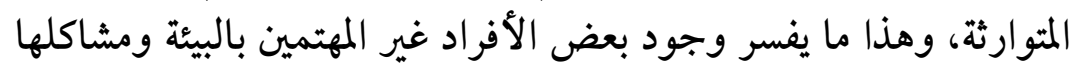

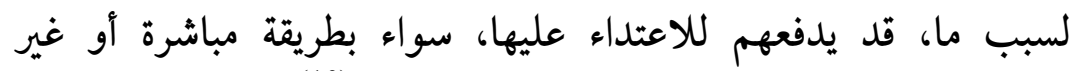

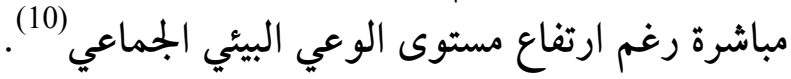
كما أن الثقافة البيئة باعتبارها أسلوب لحماية البيئة فإنها بشكل عام تأخذاعي بعدين: > الأسلوب الوقائي: وهو العمل على حدوث تلافي المشكلات البيئية والحد

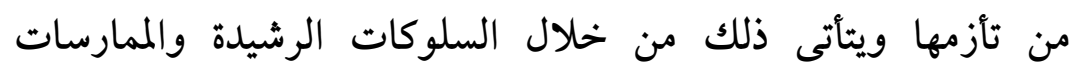

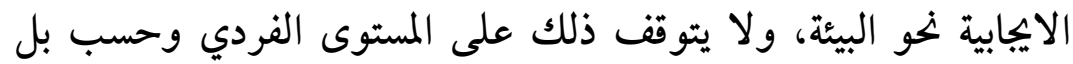


لابد أن يمس أيضا مستوى الجماعة؛ من خلال النصح، الإرشاد

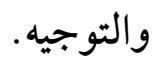

> الأسلوب العلاجي: محاولة معالجة المشكلات البيئية، بغرض التخفيف منها أو إزالتها، ويتم ذلك على المستوى الفردي والجماعي (11). كما أن للثقافة البيئية أيضا جملة من الأبعاد نذكر من بينها: ل المحافظة على المساحات الخضراء. $\checkmark$ ل المحافظة على نظافة الأحياء. $\checkmark$ ك ترشيد استهلاك الماء. ل التعريف بالنظام البيئي ومكوناته. $\checkmark$ التعريف بالمشكلات البيئية الإقليمية والعالمية.

كما يككن الإشارة هنا إلى أهم الركائز التي تقوم عليها الثقافة البيئية: ل الشعور بالمسؤولية(12)، هي شعور الفرد بالمسؤولية اتجاه بيئنه.

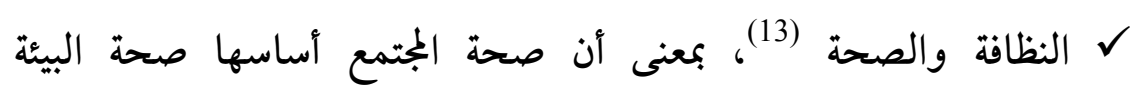

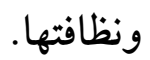

$\checkmark$ الثقافة الجمالية والذوقية (14)، بمعنى أن النظافة والذوق الفني مسؤولية لح الالتزام الشرعي (15)، دعوة الإسلام الى احترام البيئة وعدم الإضرار بها.

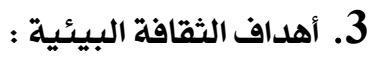
للثقافة البيئية جملة من الأهداف يمكن حصرها في النقاط التالية: إتاحة بجالات واسعة لتكوين المفاهيم وتحديد السلوكيات البيئية

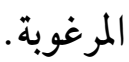


• توظيف مهارات الثفكر العليا لمواجهة التحديات البيئية.

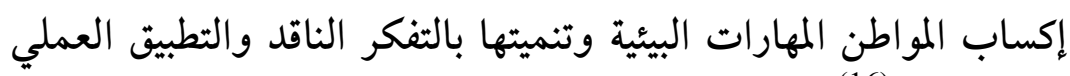

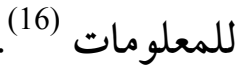

• • مماية وحفظ صحة الإنسان والبيئة على حد سواء. محاية المصادر الطبيعية كالتربة والماء والمواء والمناخ. الحفاظ على التنوع البيئي والحيوي والأماكن الطبيعية. • مماية وحفظ الموارد المعنوية والتراث الحضاري كقيم حضارية وثقافية ولئي واقتصادية للفرد والمجتمع.

استبدال المصادر الأحفورية بالمصادر الطاقوية البديلة (17). إزالة أو معالجة الأضرار البيئية القائمة. تجنب أو التقليل من المشاكل والأخطار البيئية الراهنة. الوقاية الاحتياطية من المشاكل البيئية المستقبلية والتي قد يكون من لمن الممكن تداركها (18).

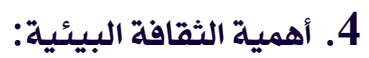

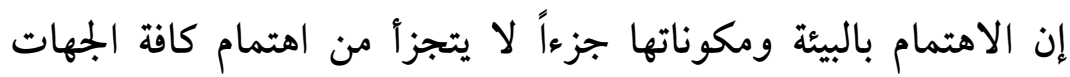

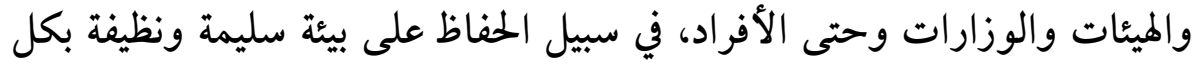

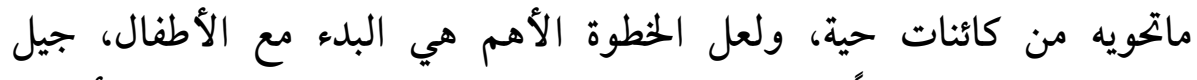

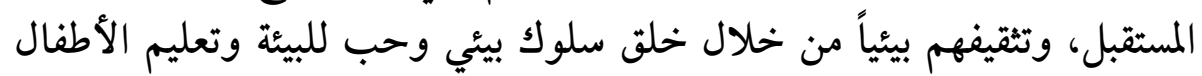

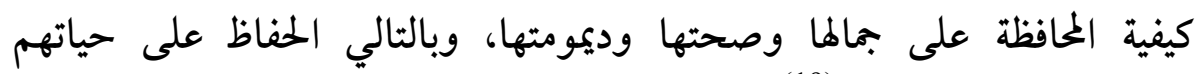
المستقبلية سليمة ومعافاة (19) ومما سبق يمكن القول بان للثقافة البيئية دور في تزويد الفرد بالمعرفة

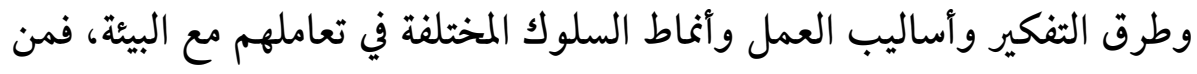


خلالها تتحقق نشأة مواطن يتمتع بالالتزام البيئي والذي يحتم عليه إتباع ماهو

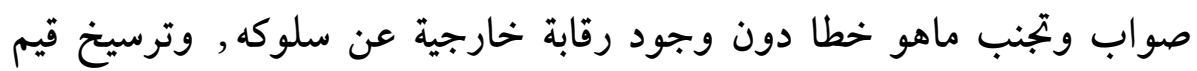

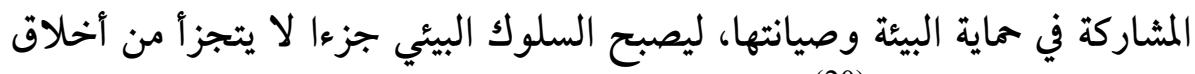
الإنسان وثقافة المجتمع (20).

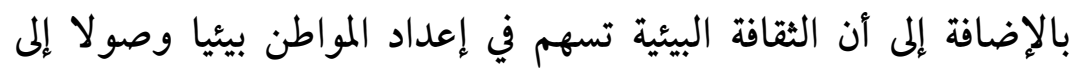

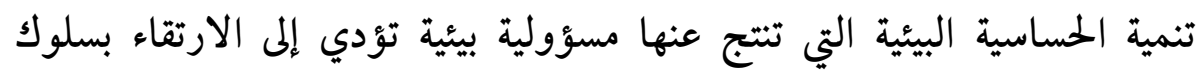

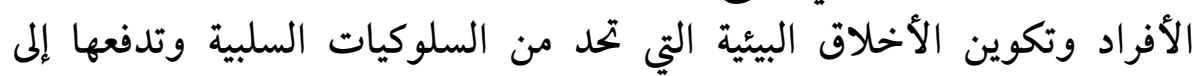
السواء (21)

الشكلات البيئية وآثارها على التنمية والصحة:

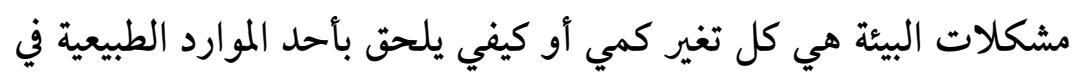

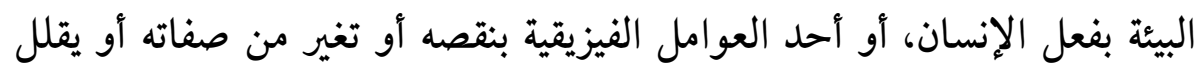

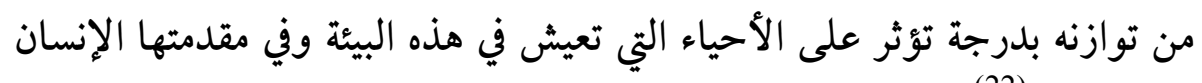
تأثيرا سلبيا (22).

إن الثقافات الإنسانية هي التي وجهت الفرد والمجتمع إلى التعامل بشكل

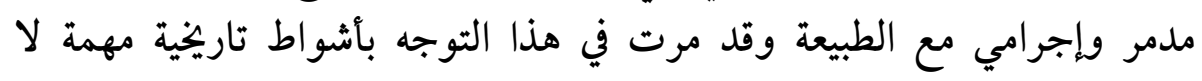

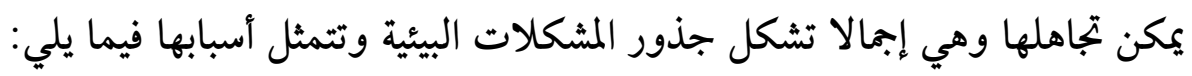

$$
\begin{aligned}
& \text { ل مسؤولية الرأسمالية تجاه البيئة. } \\
& \text { ح الصناعة والبيئة. } \\
& \checkmark
\end{aligned}
$$

$\checkmark$

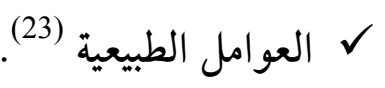


وقد أصبحت مشاكل البيئة تكتسي أهمية كبيرة على كافة المستويات من حيث أسبابها ونتائجها وكذا آثارها (24).

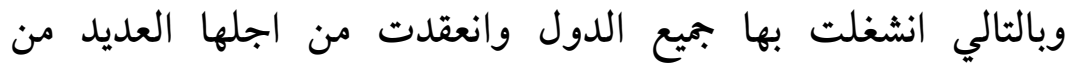

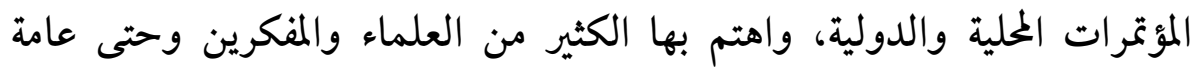
الناس (25).

6. الثقافة البيئية كأهم إستراتيجية لمعالم الشكلات البيئية:

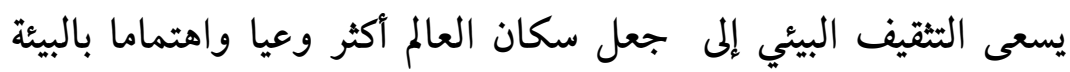

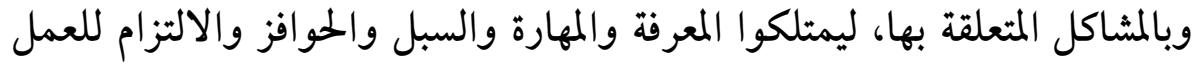

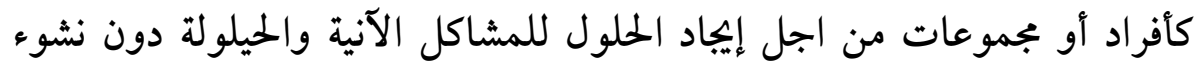

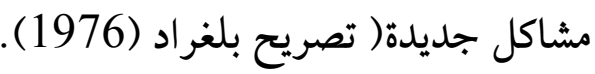

ولان علينا أن نهتم على أن يبقى على الكرة الأرضية بعد مغادرتنا لها

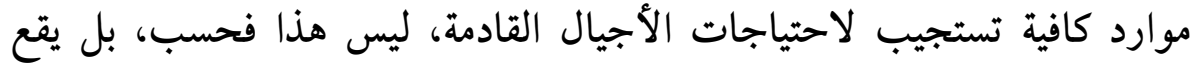

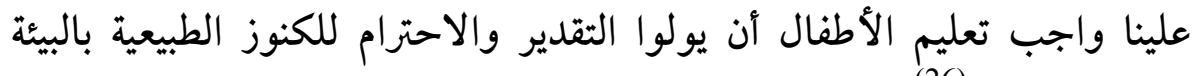
رغبة في حمايتها (26).

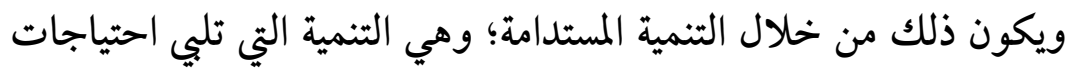

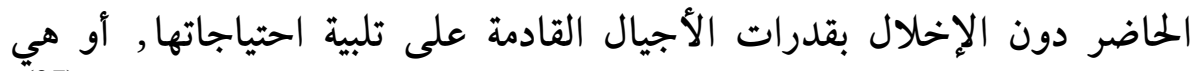

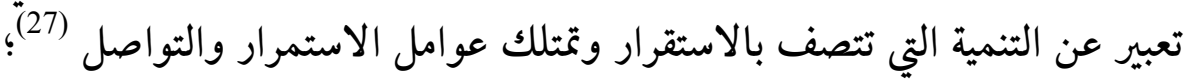

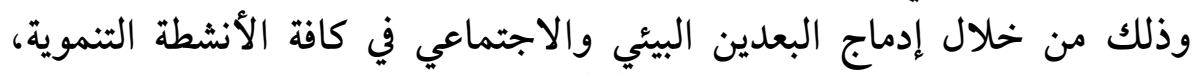
ويتم كل هذا تحت مضلة الثقافة البيئة (28).

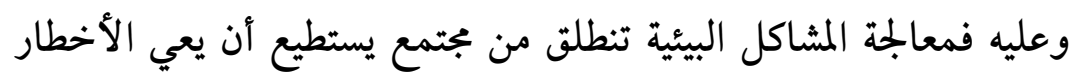

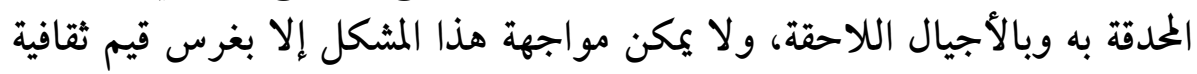

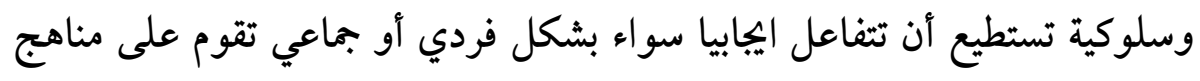

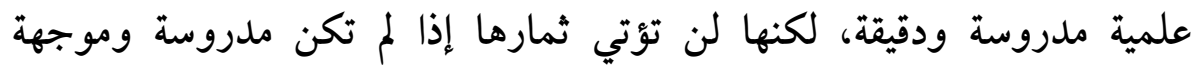

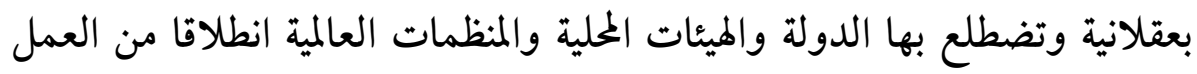




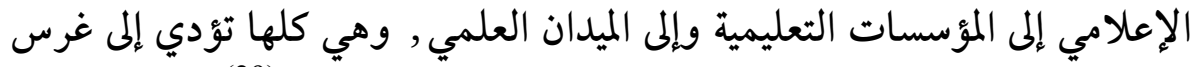
قيم جديدة وبناء أفكار تواجه السلوكات الإنسانية نحو الإييابي (29). 7.توجهات النظام التزبوي الجزائري للاهتمام بالثقافة البيئية: إن النظام التربوي هو ذلك النظام الذي يشتمل على الأدوات والمعايير

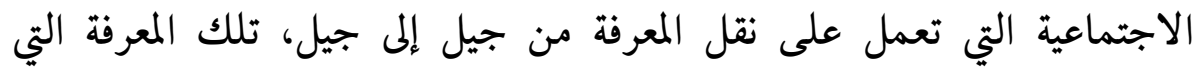

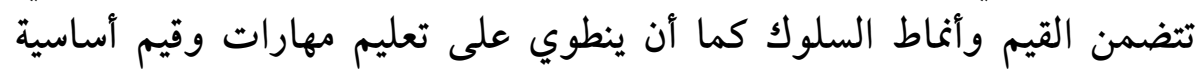
ولازمة لاستمرار المجتمع (30)

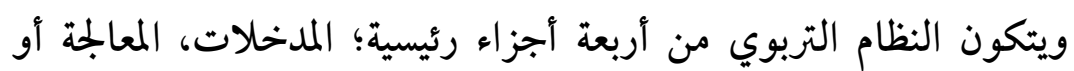

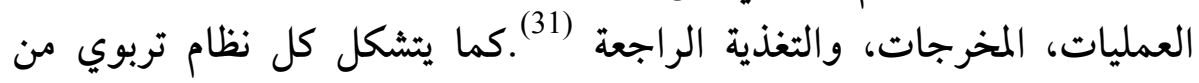

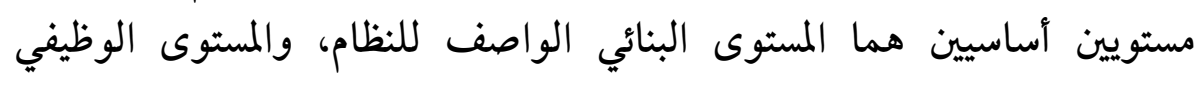

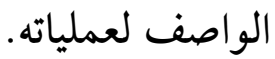

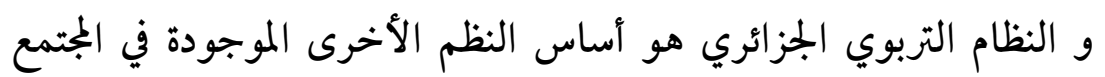

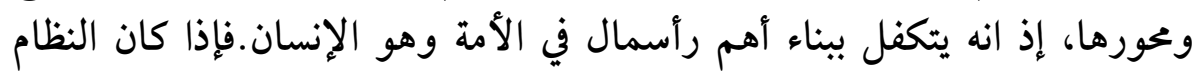

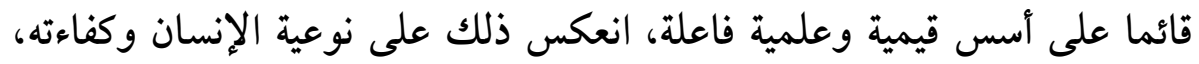

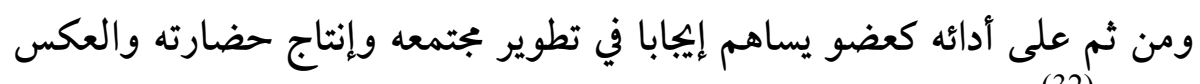

فمعالجة المشاكل البيئية تنطلق من مجتمع يستطيع أن يعي الأخطار المحدقة

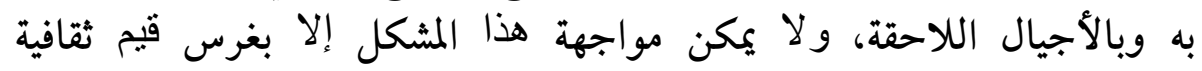

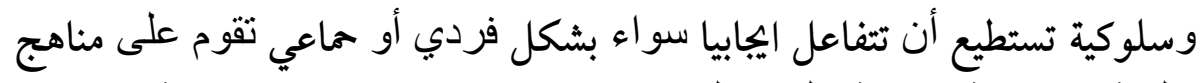

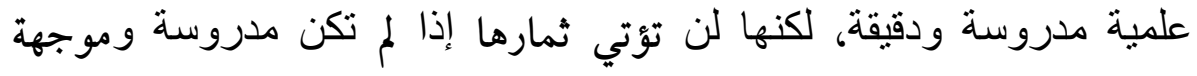

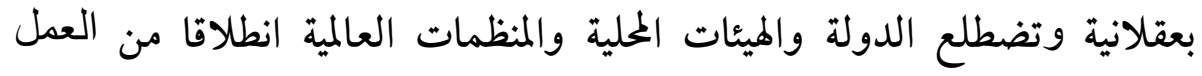

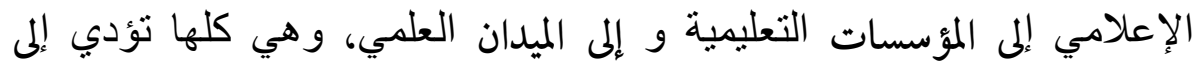
غرس قيم جديدة وبناء أفكار تواجه السلوكات الإنسانية نحو الايجابي (33). 


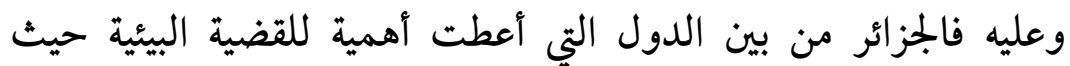

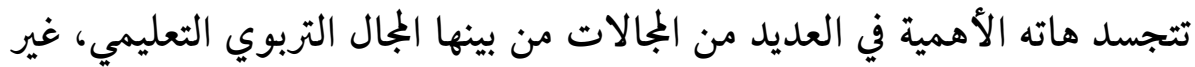

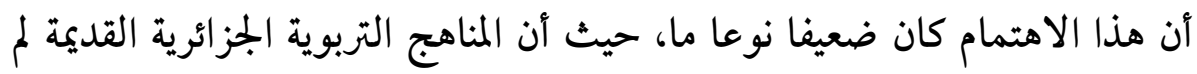

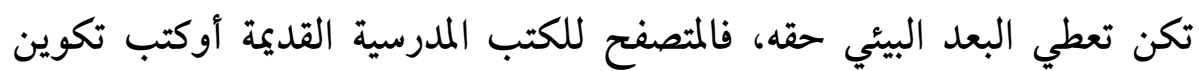

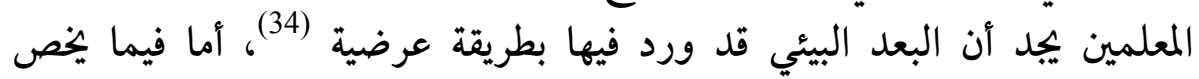

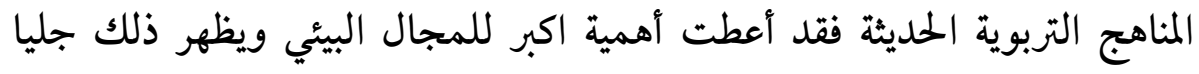

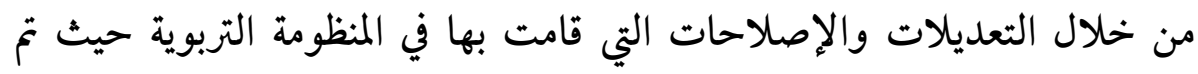

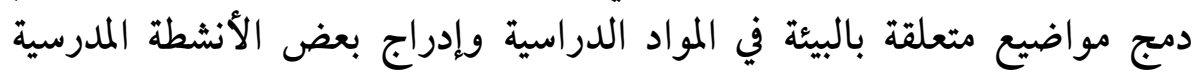

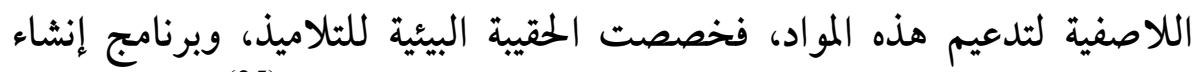

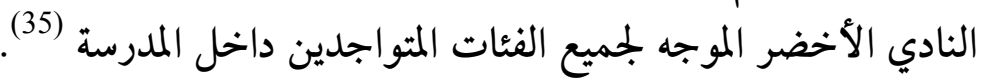

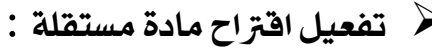

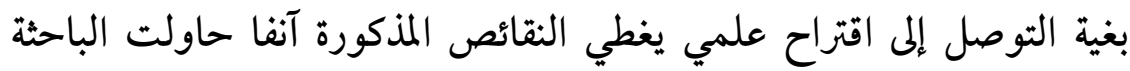

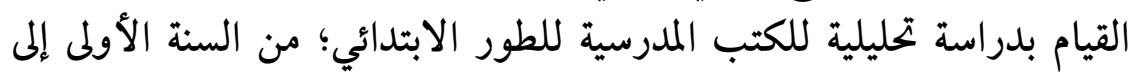
السنة الخامسة ابتدائي.

1.8 منهج الدراسة: إن طبيعـة المثـكلة والأهــاف المرسومة تفـرض على الباحث

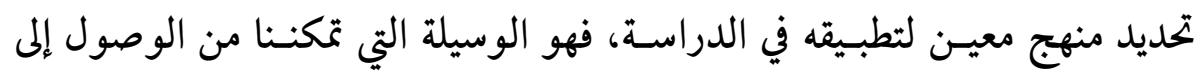

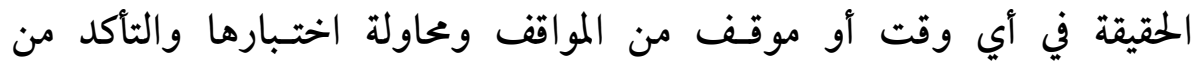

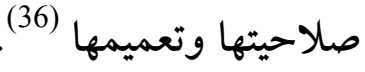

وحتى نضفي الصبغة العلمية اعتمدنا في دراستنا هذه على منهج تحليل

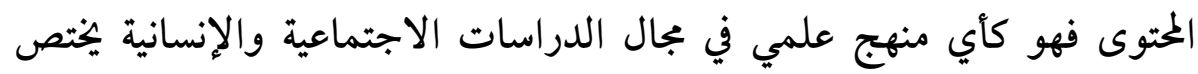

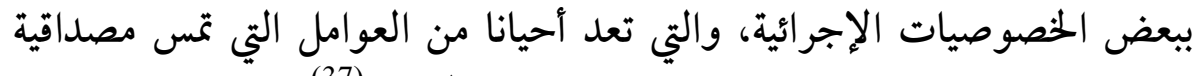
النتائج التي يخلص إليها من باب ذاتية الباحثين أو المحلين (37). 
2.8 عينة الدراسة: تعتبر العينة بجموعة فرعية من عناصر مجتمع بحث معين (38)،

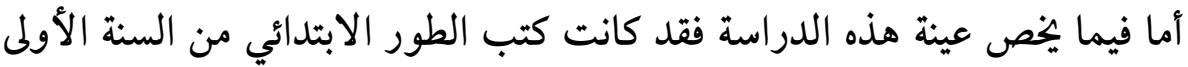

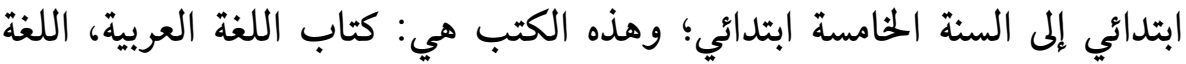

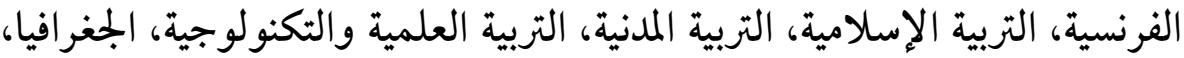
والتاريخ. 3.8 حدود الدراسة: أما فيما يخص حدود هذه الدراسة فقد كانت الكتب المدرسية للموسم الدراسي 2012/2011. 4.8 أداة الدراسة: أما فيما يتعلق بأداة الدراسة فقد كانت عبارة عن جدول يوضح؛ نسب تو افر (تناول) الكتب المدرسية لمواضيع البيئة حسب المادة الدراسية:

\begin{tabular}{|c|c|c|c|c|c|c|c|c|c|c|c|c|}
\hline م- & $\%$ & 05 & $\%$ & 04 & $\%$ & 03 & $\%$ & 02 & $\%$ & 01 & لالزينة & الرقم \\
\hline 10 & $\begin{array}{r}96.2 \\
6\end{array}$ & 03 & 87 & 03 & 100 & 01 & 77 & 02 & 67 & 01 & اللغة العربية & 1 \\
\hline 10 & 92 & 04 & 78 & 04 & 100 & 02 & /IIIIII & $\begin{array}{l}1111 \\
1\end{array}$ & |IIIIII & III & الفرنسية & 2 \\
\hline 26 & 70 & 07 & 58.06 & 07 & $\begin{array}{c}54.0 \\
5\end{array}$ & 07 & 82 & 01 & 27.27 & 04 & الإسلاميية & 3 \\
\hline 22 & 71 & 06 & 61 & 05 & 83 & 04 & 68.18 & 04 & 46 & 03 & التربية المدنية & 4 \\
\hline 10 & 125 & 01 & 100 & 01 & 96 & 03 & 74 & 03 & 63 & 02 & $\begin{array}{c}\text { التكنولوجية.عية } \\
\text { التوبية }\end{array}$ & 5 \\
\hline 10 & 100 & 02 & 100 & 02 & 56 & 06 & IIIIIII & $\begin{array}{l}1 / 11 \\
1\end{array}$ & |IIIIII & $\begin{array}{l}1 / 1 / \\
/ 1\end{array}$ & الجغرافيا & 6 \\
\hline 16 & 79 & 05 & 59.26 & 06 & 56 & 05 & /IIIIII & $\begin{array}{l}1 / 11 \\
1\end{array}$ & |IIIIII & IIII & التاريخ & 7 \\
\hline //I & //I/I/ & 28 & /I/II/I & 28 & /////I & 28 & $\mid / / / / / /$ & 10 & $\mid / / / I / /$ & 10 & & المجموع \\
\hline
\end{tabular}




\begin{tabular}{|l|l|l|l|l|l|l|l|l|l|l|l|}
\hline 1 & 1 & & & & 1 & & & & & & \\
\hline
\end{tabular}

نلاحظ من خلال الجدول المدون أعلاه أن نسبة 67 \%، 77 \%، 100 \%

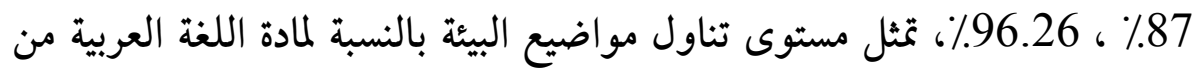
السنة الأولى إلى السنة الخامسة ابتدائي على التوالي. أما بالنسبة لمادة اللغة الفرنسية فقد كانت نسبة مستوى تناول البيئة في السنة الثالثة

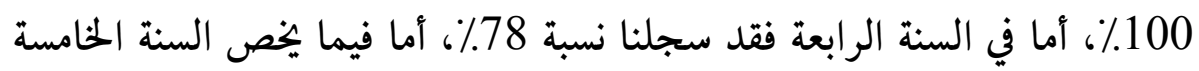

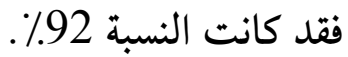

أما النسب 27.27\%، 82 \%، 54.05\%، 58.06 \% \% \% \% \%

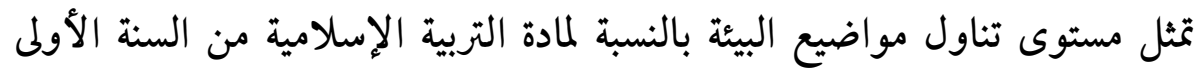

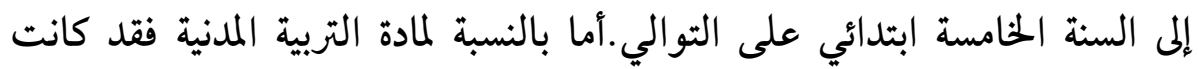

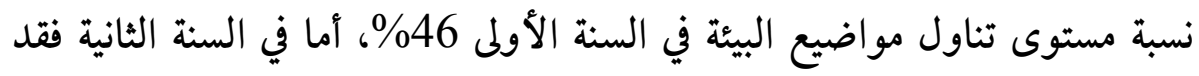
سجلنا نسبة 68.18\%، أما فيما يخص السنة الثالثة فقد كانت النسبة نجد نسبة 61 \% في السنة الرابعة، ونسبة 71 \%

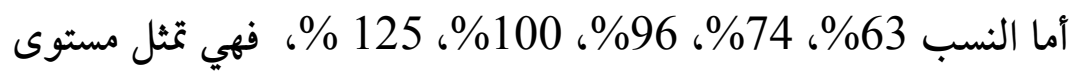

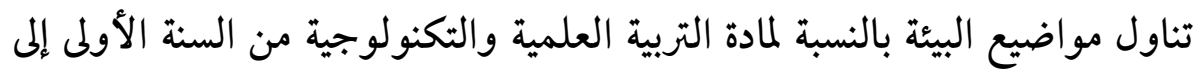

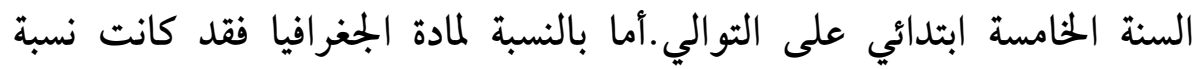

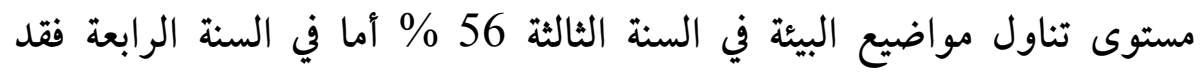

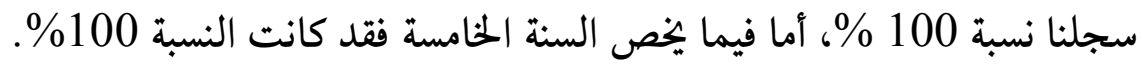
أما بالنسبة لمادة التاريخ فقد كانت نسبة مستوى تناول مواضيع البيئة في السنة الثالثة 56\%، أما في السنة الرابعة فقد سجلنا نسبة

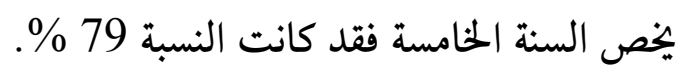
هذا فيما يخص مستوى تناول الكتب المدرسية لمواضيع البيئة حسب المادة

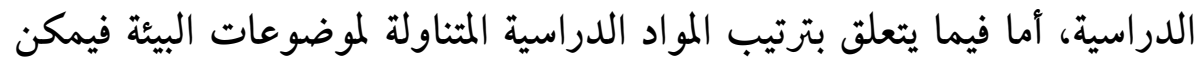


القول بان مادة اللغة العربية احتلت المرتبة 01 في السنة الأولى والثالثة، والمرتبة رقم 02 في السنة الثانية، والمرتبة رقم 03 في السنة الرابعة والخامسة.

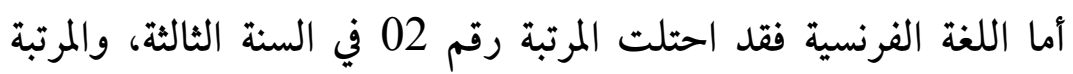

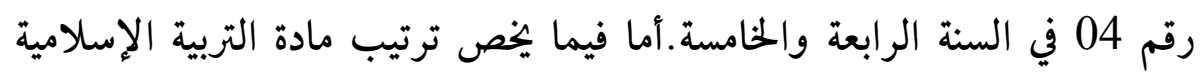

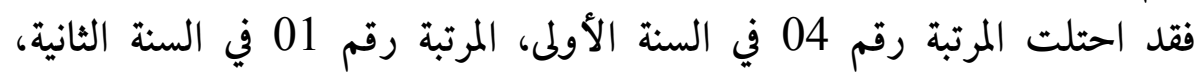
والمرتبة رقم 07 في السنة الثالثة والرابعة والخامسة.

وفيما يتعلق بترتيب مادة التربية التربية المدنية فقد احتلت المرتبة رقم 03

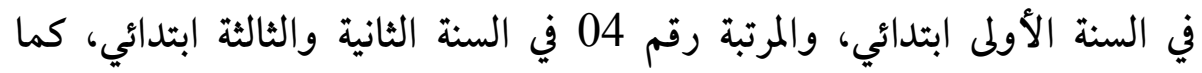

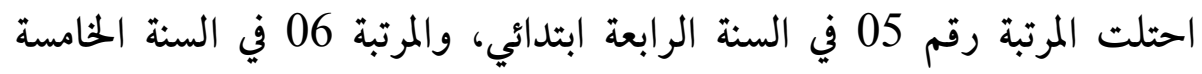

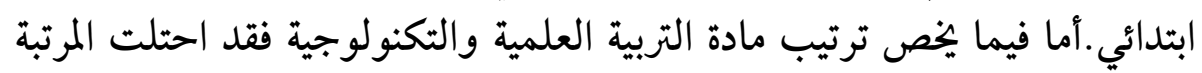

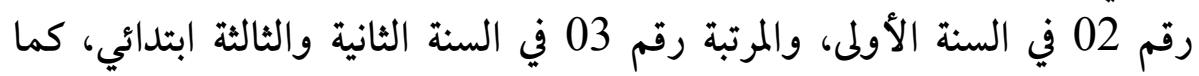
احتلت المرتبة رقم 01 في السنة الرابعة والخامسة ابتدائي.

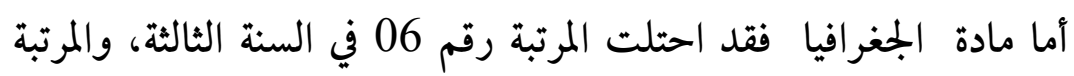

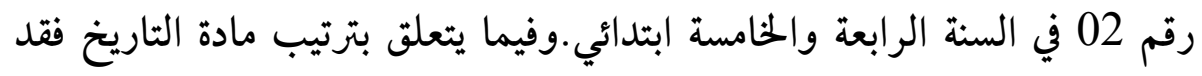

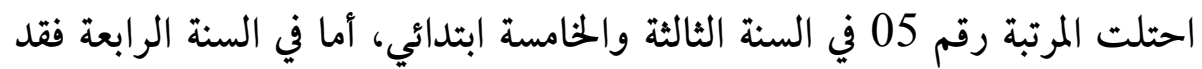

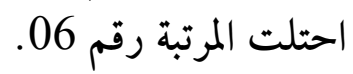

ومن خلال قراءتنا للنتائج المسجلة في الجدول أعلاه؛ اتضح لنا لنا أن تناول

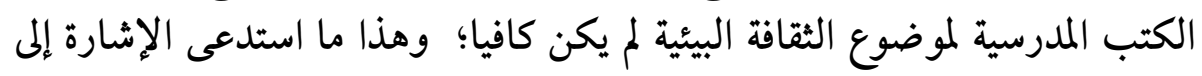

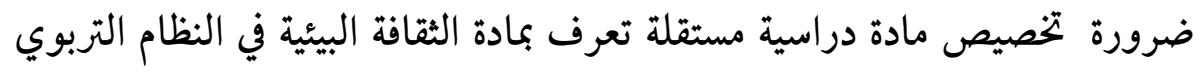

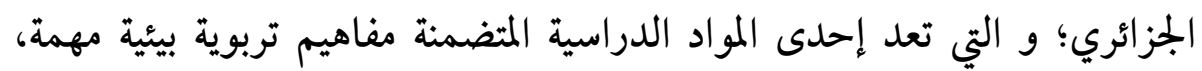

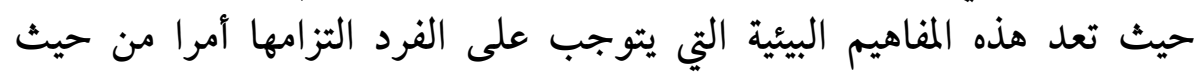

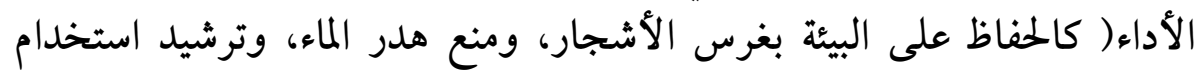

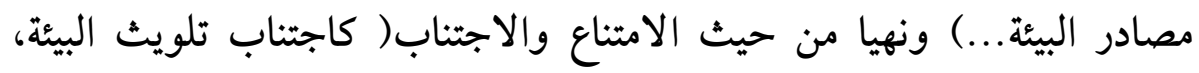

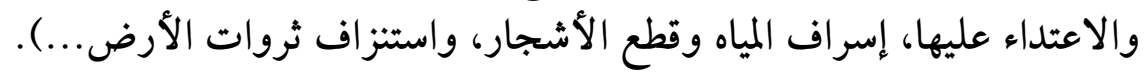


فالثقافة البيئة، هي ثقافة احترام وصحّة وذوق و مشاعر الطفل نفسه،

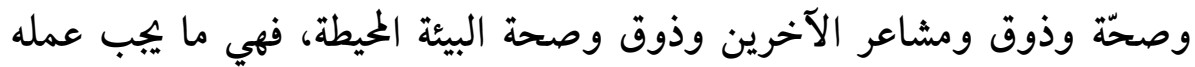

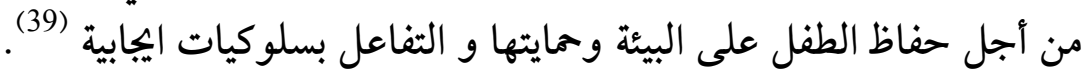

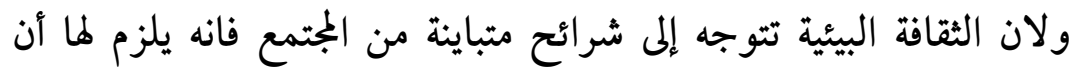

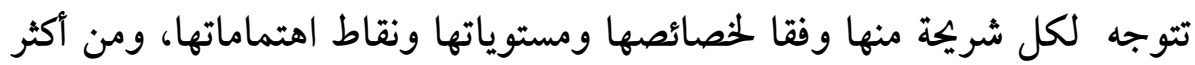

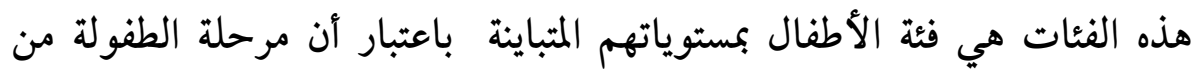

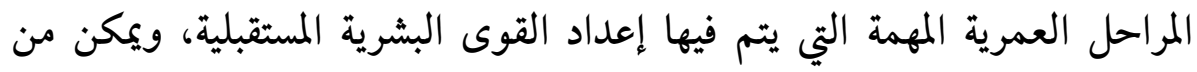

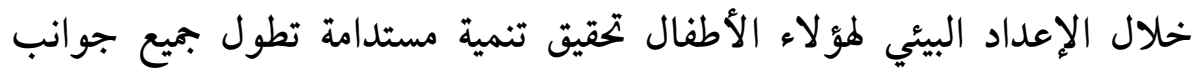

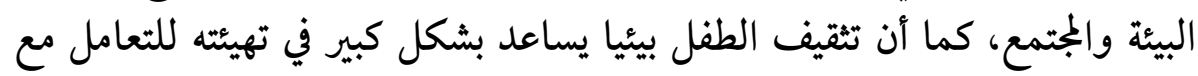

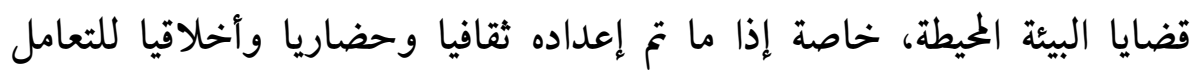

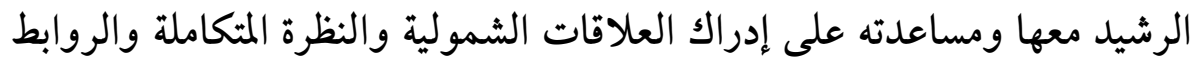

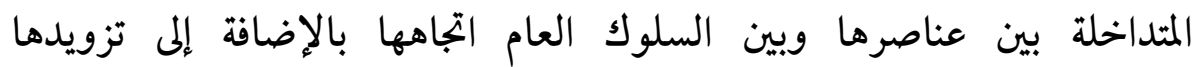
بالمعلومات البيئية المناسبة التي تجعله يسلك سلوكا بيئيا اييابيا مسؤولا (40)

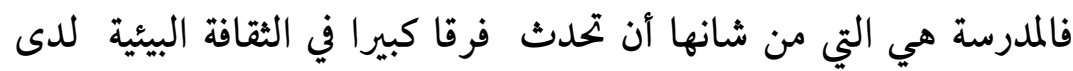

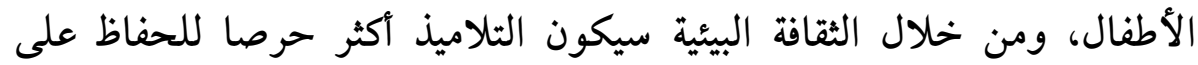

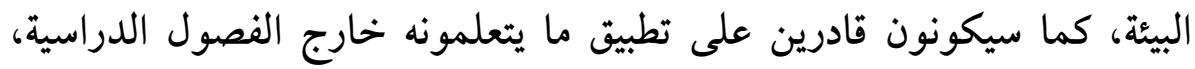

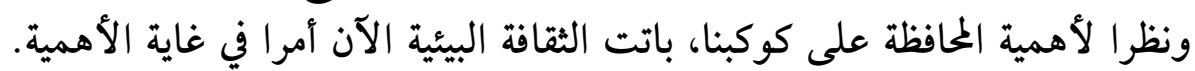

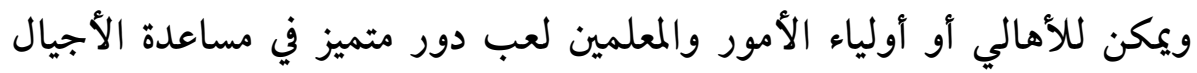

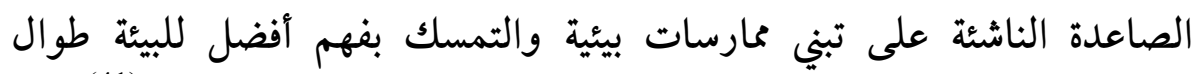
حياتهم، وتحفيزهم ليصبحوا مواطنين ذوي إحساس بالمسؤولية تجاه البيئة (41). 
خاتمةة:

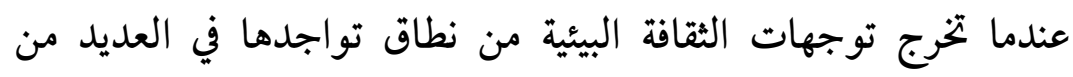

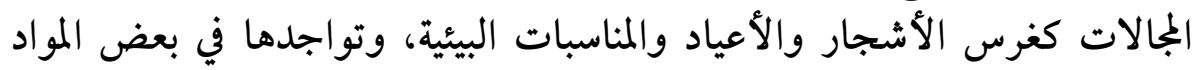

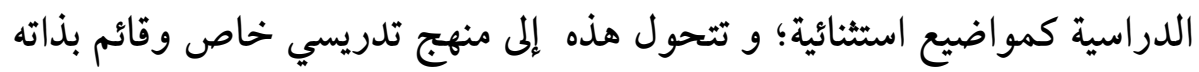

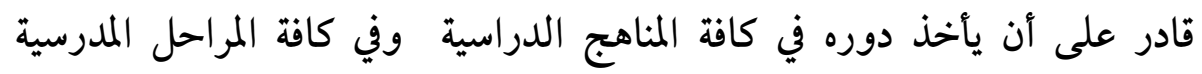

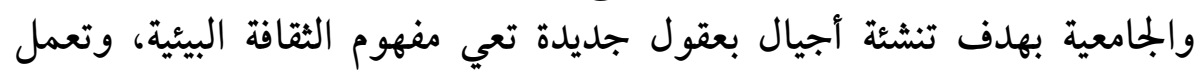

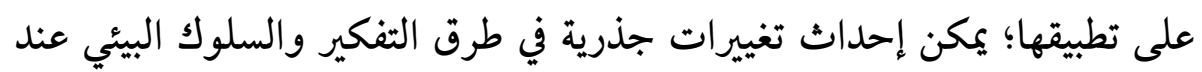

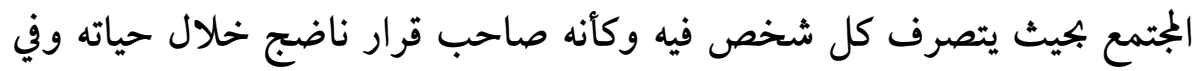
كافة نشاطاته وأعماله. 


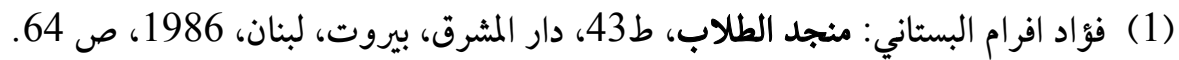
(2) فايز محمد الحديدي: ثقافة تروية- التربية مبادى و اصول-هابل، دار أسامة، عمان، الأردن، 2007، ص 156.

(3) عبد المنعم عبد القادر الميلادي، اصول التربية، مؤسسة شباب الجامعة، الإسكندرية، 2004، 204 119 ذيب فيصل: دور المسجد في نشر الثقافة البيئة- مساجد بلدية عين اعبيد نموذجا-، مذكرة مكملة لنيل شهادة الماجستير في علم الاجتماع البيئي، كلية العلوم الإنسانية والعلوم الاجتماعية، قسم علم الاجتماع، جامعة منتوري- قسنطينة-، 2008/ 2009، ص 19. (5) محمد أبو سمرة: الإعلام الزراعي والبيئ، دار الراية، عمان، الأردن، 2009، ص 15. (6) سوزان أحمد أبورية: الإنسان والبيئة والمجتمع، دار المعرفة الجامعية، الإسكندرية، مصر، 2008، ص 29

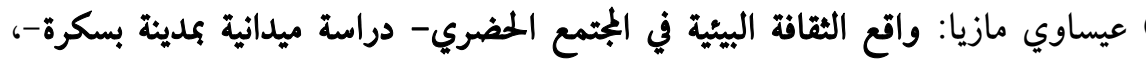

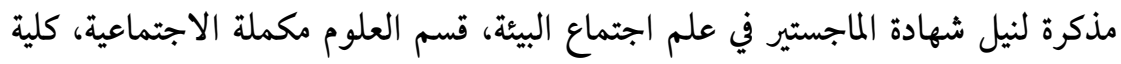

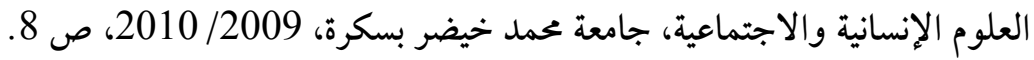

(8) شتوي الأخضر، برامج التربية البيئة في التلفزيون الجزائري- دراسة تحليلية لسلاسل

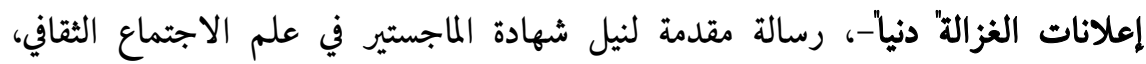

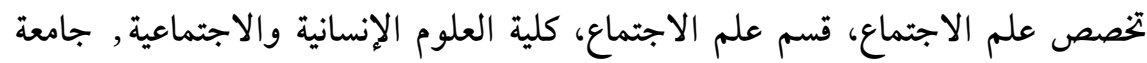

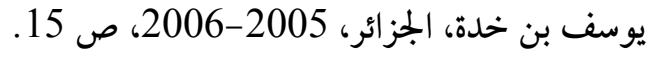


حسن محمد محي الدين السعدي: دراسات في العلوم الإنسانية وقضايا البيئة دار المعرفة

$$
\text { الجامعية، الإسكندرية، 2008، ص } 240 .
$$

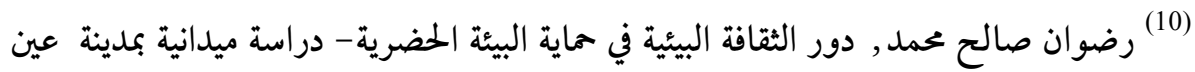

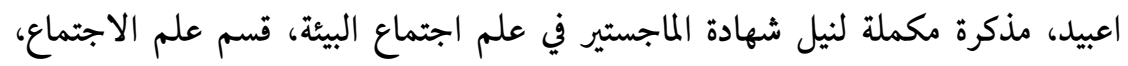

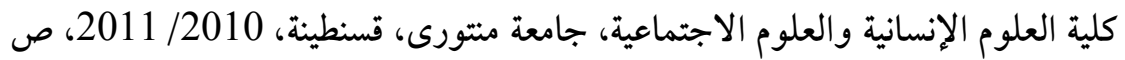

$$
\text { (11) }
$$

(12) أيمن مزاهرة، علي الشوابكة: البيئة والمجتمع، دار الشروق، عمان، الأردن، 2008، ص ص

مصطفى قمش وآخرون: مبادى الصحة العامة، دار الفكر، عمان، الأردن، 2000، ص

مزياني نور الدين، قحام وهيبة: التوعية البيئية ودورما في تحقيق التنمية المستدامة، الملتقى الوطني الخامس حول اقتصاد البيئة، كلية العلوم الاقتصادية، جامعة 20اوت 55 سكيكدة،

$$
\text { بتاريخ: 11-12 المرجع السابق، ص 4. 2008، ص } 3 .
$$

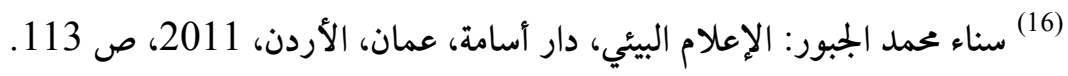

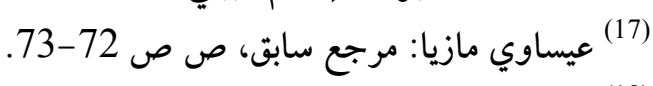

(18) تعميق الثقافة البيئية واكتشاف المواهب عنباند صند الأطفال .... مشاركة واسعة لمشروع سبانا في

$$
\text { الاحتفالية }
$$

http://fedaa.alwehda.gov.sy/_archives.asp?FileName=994787407201

00609230259، ص 02 


$$
\text { (19) }
$$

(20) رضوان صالح محمد: مرجع سبق ذكره، ص 94.

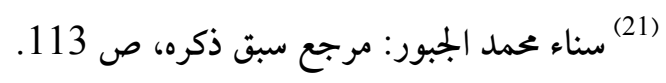

(22) ليندة شنافي: تنمية الوعي البيئي عند أفراد المجتمع، بجلة علوم الإنسان والمجتمع، كلية العلوم

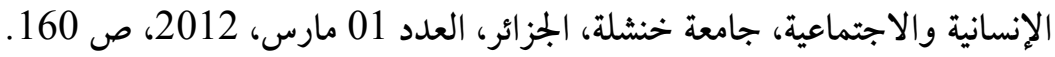
(23) أسماء مطوري: مؤسسات الشباب وحماية البيئة، مطبعة سخري، الوادي، 2012، ص ص .106-104 سامح الغراية، يحي فرحان: المدخل الى العلوم البيئية، دار الشروق، عمان، الأردن، 2011، ص 13.

(25) كمال رزيق: دور الدولة في حماية البيئة، بجلة الباحث، العدد 5، 2007، ص 95.

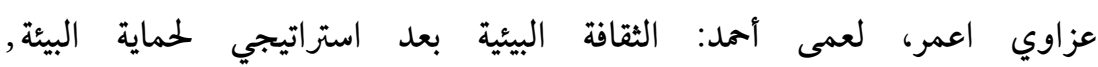

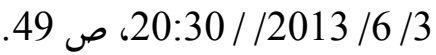
(27) خالد مصطفى قاسم: إدارة البيئة والتنمية المستدامة في ظل العولمة المعاصرة، الدار الجامعية،

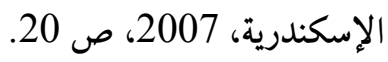

$$
\begin{aligned}
& \text { (28) عزاوي اعمر، لعمى أمد: مرجع سبق ذكره، ص } 49 . \\
& \text { المرجع السابق: ص } 50 \text { (29) }
\end{aligned}
$$

(30) محمد سلمان الخزاعلة: النظام التربوي- بين وزارتي التربية والتعليم العالي-، مكتبة المجتمع العربي، عمان، الأردن، 2010، ص 58.

$$
\text { (31) المرجع السابق: ص ص 60 61-61. }
$$

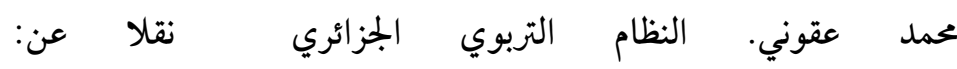
.2013/07/13/23:20 http://aladdin.7olm.org/t2986-topic 


$$
\text { عزاوي اعمر، لعمي أحمد: مرجع سبق ذكره، ص } 52 .
$$

بلعيد جمعة: دور مدارس التعليم الابتدائ والمتوسط في التربية البيئية- دراسة ميدانية

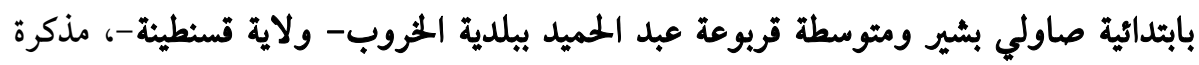

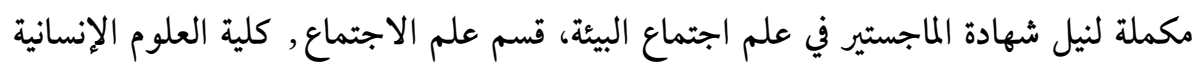

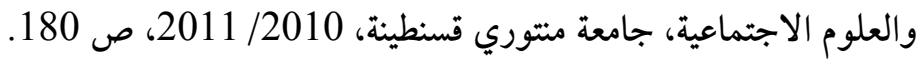

(35) عبلة غربي: التربية البيئة في المدارس الابتدائة من وجهة نظر المعلمين، مدارس مدينة

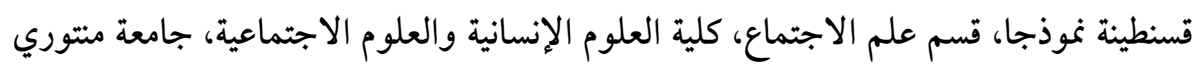

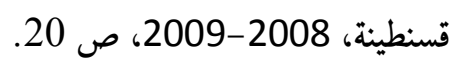

(36) فوزي غرايبة وآخرون: اساليب البحث العلمي في العلوم الاجتماعية والإنسانية، ط2، دار

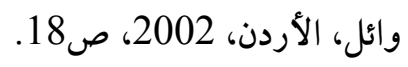

إسماعيل رابحي: الإصلاح التربوي وإشكالية الموية في المنظومة التريوية الجزائرية دراسة تحليلية تقوية لفلسفة التغيير في ضوء مقارية حل المثكل، أطروحة مقدمة لنيل درجة دكتوراه علوم في علم النفس، كلية العلوم الإنسانية والاجتماعية والعلوم الإسلامية، قسم العلوم

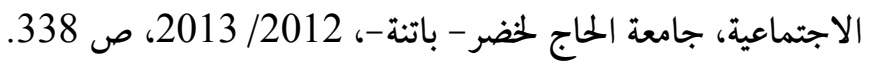

موريس أنجرس: منهجية البحث العلمي في العلوم الإنسانية تدريبات علمية، ترجمة: بوزيد

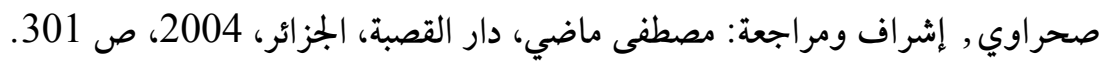
(39) وليد فاضل العبيدي: الحملة الوطنية لإعداد منامج للتربية البيئة في المدارس العراقية

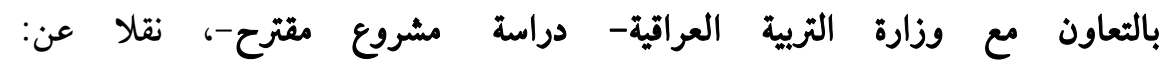
/http://www.m3arej.com

$$
\text { سناء محمد الجبور: مرجع سبق ذكره، ص } 111 .
$$

برنامج بيئتي وطني التثقيفي, يعزز الوعي البيئي بين الطلاب المدارس, نقلا عن : .ww.alittihad.ae/mobile/details.php?id=121527\&y=2012

$$
\text { .01 00:30/2013/05/13 }
$$


